\title{
Atrial Fibrillation Presenting with Acute Abdomen in an Elderly Patient - A Case Report
}

\author{
Kolluru Karthik Raja1, Shilpa Gaidhane², Sharad Bhagwanrao Sonawane ${ }^{3}$, Nazli Khatib ${ }^{4}$, Yash Gupte ${ }^{5}$ \\ ${ }^{1}$ Department of General Medicine, Jawaharlal Nehru Medical College, Datta Meghe Institute of Medical \\ Sciences (Deemed to Be University), Wardha, Maharashtra, India. ${ }^{2}$ Department of General Medicine, \\ Jawaharlal Nehru Medical College, Datta Meghe Institute of Medical Sciences (Deemed to Be University), \\ Wardha, Maharashtra, India. ${ }^{3}$ Department of General Medicine, Jawaharlal Nehru Medical College, Datta \\ Meghe Institute of Medical Sciences (Deemed to Be University), Wardha, Maharashtra, India. ${ }^{4}$ Department \\ of General Medicine, Jawaharlal Nehru Medical College, Datta Meghe Institute of Medical Sciences (Deemed \\ to Be University), Wardha, Maharashtra, India. ${ }^{5}$ Department of General Medicine, Jawaharlal Nehru Medical \\ College, Datta Meghe Institute of Medical Sciences (Deemed to Be University), Wardha, Maharashtra, India.
}

\section{PRESENTATION OF CASE}

A 63 years old male patient was admitted with complaints of acute pain in abdomen and nausea since 1 day. He had no history of chest pain, palpitations, shortness of breath, orthopnoea, paroxysmal nocturnal dyspnoea. There was no history of constipation or obstipation, vomiting's, haematuria, or oliguria. Patient had a past history of systemic hypertension, diabetes, IHD since last 8 years. He was nonsmoker, non-alcoholic. On examination, he was mesomorphic, febrile, had pulse rate of 146 bpm, irregularly irregular, his blood pressure was 134 / $86 \mathrm{mmHg}$. He had bilateral pitting oedema feet and on abdominal examination, there was diffuse tenderness in all the quadrants of abdomen. Guarding or rigidity over the abdomen was absent.

ECG was suggestive of atrial fibrillation with fast ventricular rate as shown in figure 1. Haemoglobin 12.8 gm per dL, WBC - 24,900 cells / cumm, CKMB - 6, Troponin I was negative, serum cholesterol - $95 \mathrm{mg} / \mathrm{dL}$, serum triglycerides - $91 \mathrm{mg}$ / dL, LDL - 63 mg / dL, VLDL - 18 mg / dL, HDL - 14 mg / dL, serum LDH - 382 U / L, CRP - raised, D - dimer was positive, CHA2DS2 - VASC score was 4 and HAS - BLED score was 3. In view of persistent acute pain in abdomen, he underwent CECT abdomen which was suggestive of hepatosplenomegaly with hyper dense liver with multiple splenic and renal infarcts as shown in figure 2. 2D ECHO findings were dilated cardiomyopathy with congestive cardiac failure (ischemic) with $40 \%$ left ventricular ejection fraction. He underwent coronary angiography, which revealed ischemic heart disease.

Simultaneous presentation of splenic infarction and renal infarction is a rare entity in a patient with atrial fibrillation and it is most likely due to embolization from left atria. We encountered an elderly male patient admitted with the complaints of acute pain in left upper quadrant bilateral flanks. ECG was suggestive of atrial fibrillation with fast ventricular rate. After stabilization, his CECT abdomen revealed hepatosplenomegaly with hyper dense liver and multiple infarcts in spleen and kidneys. Patient was treated as a case of solid organ infarction secondary to atrial fibrillation. Patient developed acute kidney injury over a period of 5 days and recovered after one month.

Acute simultaneous splenic infarct and bilateral renal infarcts are rare. They are associated with malignancy $30 \%$, haematological diseases $15-20 \%$, vascular $20 \%$ and Atrial fibrillation $20 \%$. AF is the most common chronic arrhythmia that can be associated with high risk of thromboembolism. ${ }^{1}$ The diagnosis of solid organ infarction is generally underdiagnosed because of the confounding signs and symptoms at the time of presentation. If the timely diagnosis of acute renal infarcts are failed to notice, there is an increased growth in mortality and morbidity in the affected patient due to acute kidney injury. ${ }^{2}$
Corresponding Author: Dr. Shilpa Gaidhane, Associate Professor, Department of General Medicine, Jawaharlal Nehru Medical College, Datta Meghe Institute of Medical Sciences (Deemed to Be University), Wardha, Maharashtra, India.

E-mail: drshilpagaidhane@gmail.com

DOI: $10.14260 / \mathrm{jemds} / 2020 / 661$

How to Cite This Article:

Raja KK, Gaidhane S, Sonawane SB, et al. Atrial fibrillation presenting with acute abdomen in an elderly patient - a case report. J Evolution Med Dent Sci 2020;9(40):3019-3021, DOI: $10.14260 / \mathrm{jemds} / 2020 / 661$

Submission 23-06-2020,

Peer Review 24-08-2020,

Acceptance 31-08-2020,

Published 05-10-2020.

Copyright (C) 2020 Kolluru Karthik Raja et al. This is an open access article distributed under Creative Commons Attribution License [Attribution 4.0 International (CC BY 4.0)] 
He was treated with heparin, dual antiplatelets, statins, beta blockers and supportive management. Patient developed uraemia due to acute kidney injury (Table 1). Patient was dialysed daily for one week following which his GFR improved.

\begin{tabular}{|ccccc|}
\hline & Blood Urea & $\begin{array}{c}\text { Serum } \\
\text { Creatinine }\end{array}$ & Sodium & Potassium \\
Day - 1 & 64 & 0.9 & 130 & 5.2 \\
Day - 3 & 110 & 2.0 & 128 & 5.6 \\
Day - 5 & 144 & 2.5 & 130 & 5.8 \\
Day - 7 & 187 & 4.2 & 134 & 5.9 \\
\hline Table 1. Renal Function Test of the Patient (Normal at the Time of \\
Admission. But, Gradually Became Deranged Indicating \\
Acute Kidney Injury) \\
\hline \multicolumn{5}{|c}{} \\
\hline
\end{tabular}
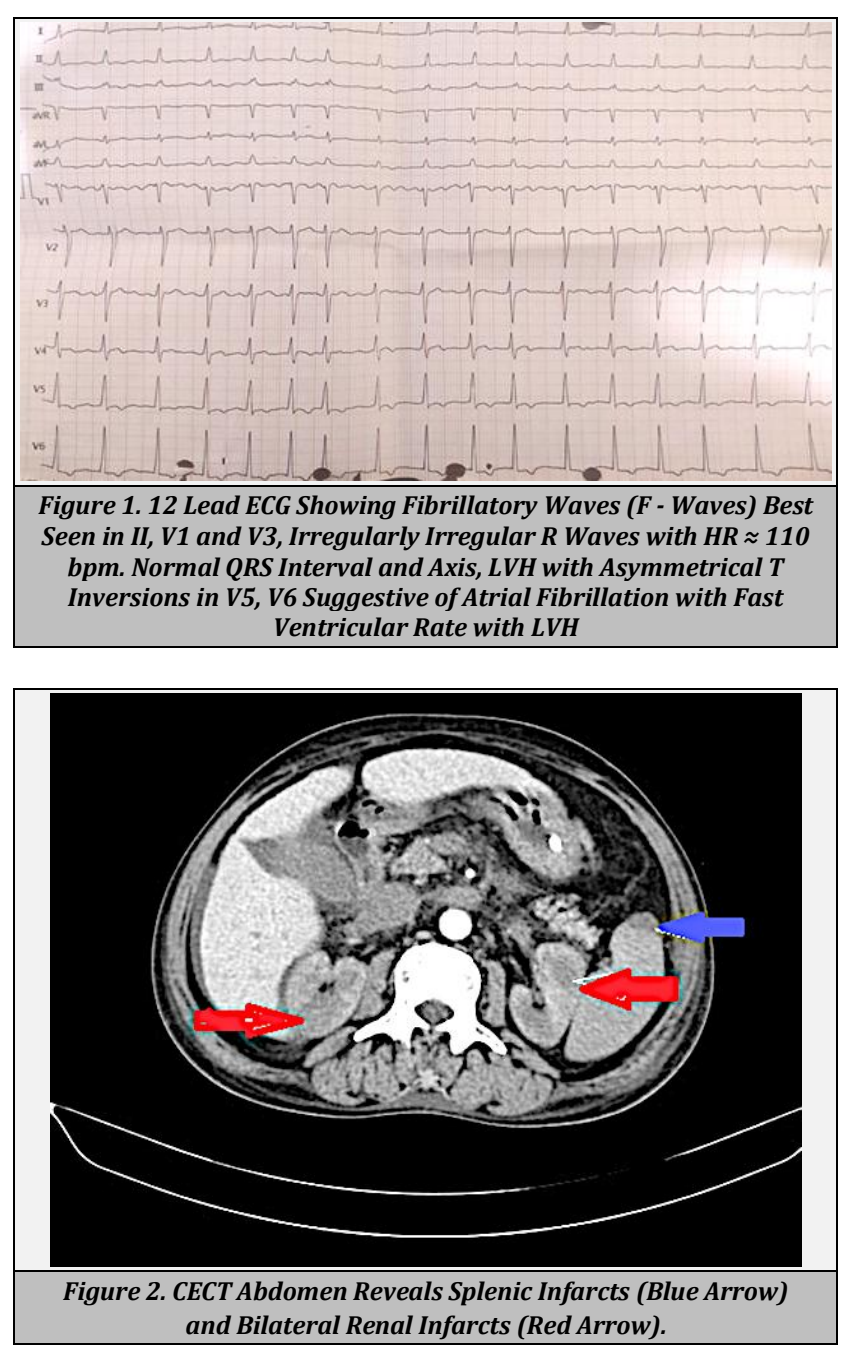

\section{DISCUSSION}

The main predisposing risk factor resulting in renal infarction is atrial fibrillation (AF). Other risk factors include systemic hypertension, IHD, mitral stenosis $^{3}$ and less commonly trauma, clotting disorders, sickle cell disease, Marfan's syndrome, vessel anomalies like fibromuscular dysplasia. Narcotics like marijuana or cocaine have also been involved and sometimes idiopathic.

Renal infarcts is mainly seen in elderly. ${ }^{4}$ Patients usually presents with bilateral flank pain / pain in epigastrium region accompanied with nausea and vomiting. Patient may have haematuria and fever is documented in the half the cases presenting with renal infarcts. The symptoms of acute renal infarct (ARI) commonly mimics other diagnosis like - "urinary tract infection (UTI), nephrolithiasis, biliary pathogenesis, appendicitis and mesenteric ischemia". So often the diagnosis acute renal infarct due to atrial fibrillation is under diagnosed. ${ }^{5}$

Lab investigations reveals leucocytosis, deranged lipid profile, deranged liver function tests, urine routine and microscopy shows haematuria and proteinuria. ${ }^{5}$ The Absence of haematuria in our case correlated with the marked decline in blood flow to the infarcted region within the kidney. ${ }^{3}$ Rise of lactate dehydrogenase (LDH) is believed to be most sensitive characteristic feature of acute renal infarct. ARI can be diagnosed by CECT abdomen and pelvis, renal arteriography. The gold standard investigation for diagnosing renal infarcts is renal arteriography. In view of raised blood urea and serum creatinine levels, renal arteriography was not done in our patient. CECT abdomen and pelvis is the widely accepted diagnostic tool of choice in acute abdomen. 5 'Cortex corticis' or 'Cortical rim sign' is the radiological finding on CT imaging.

Complications of bilateral renal infarction include systemic hypertension, renal artery dissection (fibromuscular dysplasia or atherosclerosis), acute kidney injury, chronic kidney disease.

Treatment of choice of renal infarction is anticoagulation. Intravenous heparin overlapped with oral anticoagulants (Warfarin). ${ }^{4}$ Target of INR is 2.5 to $3.5 .^{3}$ Thrombolysis can be done in patients who are diagnosed early within 90 to 180 minutes. Medical management showed better results than surgical management in a case of unilateral artery embolism. Open surgery is only recommended in trauma. "Local thrombolysis or thrombectomy with percutaneous endovascular therapy" is usually performed for occlusion in the chief renal artery or substantial branches. Some patient may need dialysis (approximately 8 percent in a case series). ${ }^{4}$

Splenic infarction results in tissue necrosis due to interruption of blood supply. The common cause of splenic infarction is haematological malignancies (chronic myeloid leukaemia and myelofibrosis). ${ }^{6}$ It also develops in cardiovascular disorders, autoimmune disorders, trauma, infection, surgery. Symptoms include non-specific pain and tenderness in the abdomen mainly in the left hypochondrium. It may also accompanied with nausea, vomiting, fever. There is no specific laboratory test for diagnosing splenic infarction. Leucocytosis is common finding in a peripheral smear. ${ }^{7}$ As reported in a literature D-dimer was strongly correlated with splenic infarction. ${ }^{8}$ This outcome contemplate that aetiology is secondary to thromboembolism.

The diagnosis of splenic infarction depends on both clinical scenario \& imaging considerations. CECT abdomen is best radiological imaging for the diagnosis. Angio-computed tomography is the demonstration methodology of decision. Ultrasonography and conventional radiology are helpful in the differential determination with other abdominal and thoracic diseases impersonating splenic infarction. ${ }^{9}$

Splenic infarcts are managed conservatively in asymptomatic or in patients without any complications. Conservative approach usually includes analgesics, antibiotics, vaccination (pneumococcal, meningococcal, Haemophilus influenza) and supportive therapy. Surgery is the treatment of choice in patients with complications due to splenic infarction like splenic abscess, splenic rupture, and pseudocyst. In case 
of complications, it is important to perform splenectomy as early as possible. ${ }^{10}$

\section{CONCLUSIONS}

Renal infarcts and splenic infarcts are uncommon in patients with atrial fibrillation. High index of suspicion for solid organ infarct is required for early diagnosis of atrial fibrillation in patients presenting with acute abdomen. Solid organ infarction mainly involving spleen and kidney may be the cause of acute abdomen in atrial fibrillation.

Financial or Other Competing Interests: None.

\section{REFERENCES}

[1] Wolf PA, Abbott RD, Kannel WB. Atrial fibrillation as an independent risk factor for stroke: the Framingham study. Stroke 1991;22(8):983-8.

[2] Hoxie HJ, Coggin CB. Renal infarction: statistical study of two hundred and five cases and detailed report of an unusual case. Arch Intern Med 1940;65(3):587-94.
[3] Domanovits H, Paulis M, Nikfardjam M, et al. Acute renal infarction. Clinical characteristics of 17 patients. Medicine (Baltimore) 1999;78(6):386-94.

[4] Lopez VM, Glauser J. A case of renal artery thrombosis with renal infarction. J Emerg Trauma Shock 2010;3(3):302.

[5] Hazanov N, Somin M, Attali M, et al. Acute renal embolism. Forty-four cases of renal infarction in patients with atrial fibrillation. Medicine (Baltimore) 2004;83(5):292-9.

[6] Nores M, Phillips EH, Morgenstern L, et al. The clinical spectrum of splenic infarction. Am Surg 1998;64(2):1828.

[7] Jaroch MT, Broughan TA, Hermann RE. The natural history of splenic infarction. Surgery 1986;100(4):74350.

[8] Hara T, Koji Y, Massive splenic infarction due to left ventricular apical thrombus in a patient with giant splenomegaly. Heart Asia 2012;4(1):53.

[9] Goerg C, Schwerk WB. Splenic infarction: sonographic patterns, diagnosis, follow-up, and complications. Radiology 1990;174(3 Pt 1):803-7.

[10] Peppas G, Fragoulis KN, Falagas ME. Successful conservative management of large splenic infarct masquerading as infection. J Gastroenterol Hepatol 2007;22(2):282-3. 